\title{
Feature Extraction of Thyroid Nodule US Images Using GLCM
}

\author{
Priti Shivaji Dhaygude ${ }^{1}$, S. M. Handore ${ }^{2}$ \\ ${ }^{1}$ Trinity COE \& Research, Pune, Maharashtra, India \\ ${ }^{2}$ Trinity COE \& Research, Pune, Maharashtra, India
}

\begin{abstract}
The Thyroid gland has a butterfly shape and is composed of two cone-like lobes. Thyroid gland belongs to the endocrine system. It is located in the neck just in front of the larynx. It controls the secretion of the thyroid hormone that regulates the temperature of the human body, and greatly affects childhood intelligence, growth, and adult metabolism. Too much or too little thyroid hormone (due to a thyroid that is too large or two small, respectively) causes pathological changes. Different methods that are used to identify and classify abnormalities of the thyroid gland include Ultrasound imaging (US), Computer Tomography (CT), Computer Aided Diagnosis (CAD), Magnetic Resonance Imaging (MRI). Ultrasound (US) imaging is mostly used popular diagnostic tool. It is inexpensive and easy to use also it may follow anatomical deformations in real time during biopsy and treatment as well as it is noninvasive and does not require ionizing radiation.
\end{abstract}

Keywords: CAD, US, ANN, GLCM, FNAC, feature extraction, segmentation

\section{Introduction}

The thyroid keeps metabolism under control through the action of thyroid hormone. It produces harmones that performs regulation of growth and rate of function of many other systems. These harmones are named as triiodothyronine and thyroxine. There are various thyroid disorders like Hypothyroidism, Hyperthyroidism, goiter and thyroid nodules (benign/malignant). Medical image analysis plays an important role in many clinical procedures for detecting different types of human diseases. Thyroid medical images are utilized for the diagnosis process

Image processing is one of the type of signal processing which uses input as an image such as photograph or video frame and gives output as an image or parameters related to the image. Medical imaging is the technique used to create images of the human body for clinical purpose. Techniques to process US images are continuously being developed. Several methods for segmenting anatomical objects from US images have been presented, such as those for segmenting the prostate [18], [19], tumors in the breast [20], the carotid artery [21], [23] and the thyroid nodule [22]. Ultrasound provides a safe tool for disease surveillance [1] Medical imaging includes the study of normal anatomy and physiology. For diagnosing thyroid diseases, Ultrasound (US) . US imaging is inexpensive, non-invasive and easy to use. US images are often preferred due to their costeffectiveness and portability in smaller hospitals. Ultrasound study of thyroid is preferred because of its superficial location, size and echogenicity obtaining the volumes of thyroid tissues to increase reliability as well as to reduce invasive operations like biopsy and Fine Needle Aspiration (FNA). Computerized system is a valuable and beneficial tool for feature extraction and classification of thyroid dule in order to eliminate operator dependence and to improve the diagnostic accuracy[16].

\section{Proposed Methodology}

Fig.1 represents the schematic of proposed method. First, US image of thyroid is taken which contains speckle noise. This noise must be removed before going to the next steps of the methodology.

Filtering-The noise in the image can be removed using linear as well as non-linear filters. Nonlinear filters are becoming more important in image processing applications. They are often better than linear filters at removing noise without distorting image features [2].It includes Median filtering as the most common method of clearing image noise. Median filter clears the noise in the image while retaining edges of an image [3].

Histogram Equalization-This process spreads out intensity values over the total range of values in order to achieve higher contrast. This process is mainly useful when we represent an by close contrast values like images in which both the background as well as foreground are bright at the same time or both are dark at the same time.

Normalization -It changes the range of pixel intensity values. The purpose of normalization is to bring the image in a range which is more familiar or normal to the senses. 


\section{International Journal of Science and Research (IJSR) \\ ISSN (Online): 2319-7064}

Index Copernicus Value (2013): 6.14 | Impact Factor (2014): 5.611

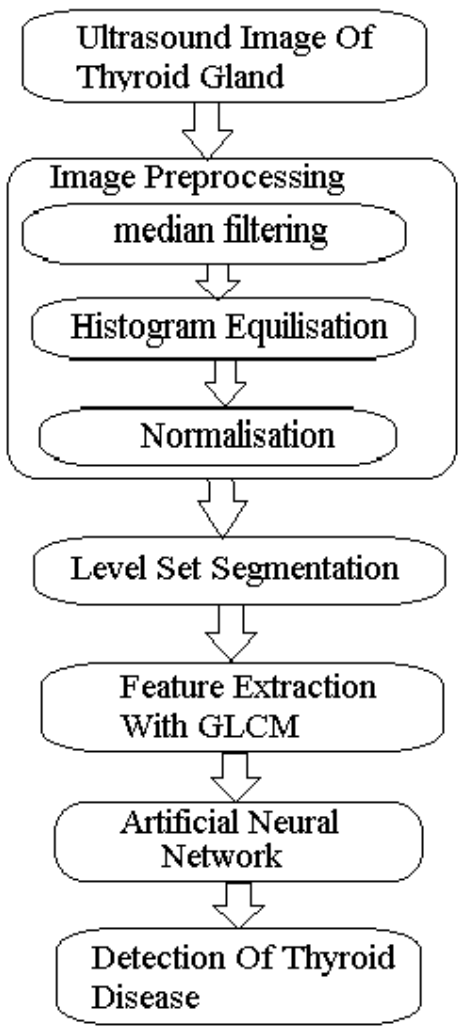

Figure 1: Schematic of Proposed Methodology

Segmentation-A process of partitioning a digital image into multiple segments is referred as image segmentation. In order to simplify and or change the representation of an image into something that is more meaningful as well as easy to analyze is the goal of segmentation[6].The level set methods have been documented to obtain good results from medical images having the boundary of the regions of interest usually have low curvature values [4][5]. With the help of level set method we can do segmentation even if there is intensity inhomogenity. Level Set Method gives numerical technique for tracking interfaces as well as shapes. The main advantages of level set method are that we can perform numerical computations including curves and surfaces [6].

Feature Extraction- It is the process of acquiring higher level information of an image like colour, shape, texture etc. Features contain the relevant information of an image. Features are divided into different classes based on the kind of properties they describe. Texture relates mostly to a specific and spatially repetitive structure of surfaces which is formed by repeating a particular element or some elements in different relative spatial positions. Texture is an important characteristic which is used in identifying regions of interest in an image. The earliest methods for texture feature Extraction is Grey Level Co-occurrence Matrices (GLCM) [7].GLCM characterize the texture of an image by calculating how often pairs of pixel with specific values and in a particular spatial relationship occur in an image. Then it creates a matrics and extracts statistical measures from this matrix.

ANN Classifier-ANN is a parallel distributed processor that has a natural tendency for storing experiential knowledge The use of textural features in ANN helps to resolve misclassification. The main benefit of neural networks is that a model of the system can be built from the available data. Classification of image is done using neural networks is done by texture feature extraction and then applying the back propagation algorithm [8].

The remaining paper is organized as follows. Section 3 covers Texture feature extraction using GLCM, Section 4 gives experimental results, Section 5 presents the conclusion and in Section 6, the references are given.

\section{Texture Feature Extraction Using GLCM}

Texture is a most significant feature of an image is widely used in medical image analysis, automatic visual inspection, image classification and remote sensing[9][10][11]. Textures are complex visual patterns consisting of entities, or subpatterns having characteristic brightness, color, slope, size, etc. A basic stage to obtain such features through texture analysis process is texture feature extraction. Texture features can be extracted with number of methods, using structural, statistical, model-based and transform information, in which a well-known method is using a Gray Level Co-occurrence Matrix (GLCM)[12]. GLCM contains the second-order statistical information of spatial relationship of image pixels. From GLCM, Haralick introduced 13 common statistical features, known as Haralick texture features. Texture is a visual pattern with properties of homogeneity which do not result from the presence of only a single color such as clouds and water [13]. Haralick suggested the use of gray level cooccurrence matrix. It always considers the relationship between two neighboring pixels, out of which the first pixel is known as a reference whereas the second is known as a neighbour pixel. GLCM matrix can be explained with the example illustrated in fig 2 for four different gray levels. Here we have used one pixel offset (a reference pixel and its immediate neighbour). When the window is large then using a larger offset is possible. In this case, the top left cell will be filled with the number of times the combination of 0,0 occurs, i.e. how many times in the image area a pixel with grey level 0 (neighbour pixel) appears to the right of another pixel with grey level 0 (reference pixel)[15]

\begin{tabular}{|l|l|l|l|l|}
\hline $\begin{array}{l}\text { neighbour pixel value }-\cdots \\
\text { ref pixel value: }\end{array}$ & 0 & 1 & 2 & 3 \\
\hline \hline 0 & 0,0 & 0,1 & 0,2 & 0,3 \\
\hline \hline 1 & 1,0 & 1,1 & 1,2 & 1,3 \\
\hline 2 & 2,0 & 2,1 & 2,2 & 2,3 \\
\hline 3 & 3,0 & 3,1 & 3,2 & 3,3 \\
\hline
\end{tabular}

Figure 2: GLCM calculation

\section{Experimental Results}

Total 16 images were used amongst which 8 images were benign (non-cancerous) and 8 images were malignant (cancerous).Total 7 features were extracted using GLCM. According to features matrices there are seven type of features which is helpful to exacts the features of images or texture of images. These following features are calculated: 


\section{International Journal of Science and Research (IJSR) \\ ISSN (Online): 2319-7064}

Index Copernicus Value (2013): 6.14 | Impact Factor (2014): 5.611

Table 1: Some features with their formulae

\begin{tabular}{|c|c|}
\hline Features & Formula \\
\hline AP & Area/Perimeter \\
\hline compactness & (Perimeter) ${ }^{2} /$ Area \\
\hline Solidity & area/convex area \\
\hline Eccentricity & Minor Axis Length / Major Axis Length \\
\hline
\end{tabular}

Table 2: Extracted Feature values of Benign Thyroid Images

\begin{tabular}{|c|c|c|c|c|}
\hline \multirow{2}{*}{ US Image } & \multicolumn{4}{|c|}{ Benign Measurement Indices } \\
\cline { 2 - 5 } & Area & Perimeter & AP & Compactness \\
\hline Case 1 & 277.25 & 115 & 2.41 & 47.7006 \\
\hline Case 2 & $7.03 \mathrm{E}+03$ & $1.03 \mathrm{E}+03$ & 6.83 & 150.588 \\
\hline Case 3 & 136 & 55.5 & 2.45 & 22.648 \\
\hline Case 4 & 10093 & $1.62 \mathrm{E}+03$ & 6.24 & 258.979 \\
\hline Case 5 & $2.18 \mathrm{E}+03$ & 564 & 3.86 & 145.74 \\
\hline Case 6 & 440.75 & 142.5 & 3.09 & 46.072 \\
\hline Case 7 & $2.44 \mathrm{E}+03$ & $4.73 \mathrm{E}+02$ & 5.17 & 91.422 \\
\hline Case 8 & $1.21 \mathrm{E}+04$ & 892.5 & 13.6 & 65.589 \\
\hline
\end{tabular}

\begin{tabular}{|c|c|c|c|}
\hline \multirow{2}{*}{ US Image } & \multicolumn{3}{|c|}{ Benign Measurement Indices } \\
\cline { 2 - 4 } & Solidity & Eccentricity & Orientation \\
\hline Case 1 & 0.6539 & 0.7626 & 17.8293 \\
\hline Case 2 & 0.5569 & 0.6992 & 13.966 \\
\hline Case 3 & 0.9306 & 0.6387 & -53.6185 \\
\hline Case 4 & 0.6344 & 0.8986 & -83.0916 \\
\hline Case 5 & 0.7745 & 0.7093 & -6.6006 \\
\hline Case 6 & 0.8041 & 0.8323 & 11.6209 \\
\hline Case 7 & 0.7612 & 0.9918 & 14.7327 \\
\hline Case 8 & 0.7785 & 0.8434 & 9.4388 \\
\hline
\end{tabular}

Table 4: Extracted Feature values of Malignant Thyroid Images

\begin{tabular}{|c|c|c|c|c|}
\hline \multirow{2}{*}{$\begin{array}{c}\text { US } \\
\text { Image }\end{array}$} & \multicolumn{4}{|c|}{ Malignant Measurement Indices } \\
\cline { 2 - 5 } Case 1 & $4.20 \mathrm{E}+03$ & 773.75 & 5.43 & 142.472 \\
\hline Case 2 & $1.01 \mathrm{E}+04$ & $1.19 \mathrm{E}+03$ & 8.43 & 141.507 \\
\hline Case 3 & $1.47 \mathrm{E}+04$ & 1318 & 11.17 & 117.936 \\
\hline Case 4 & 3614 & 669 & 5.402 & 123.84 \\
\hline Case 5 & $2.48 \mathrm{E}+03$ & 670 & 3.703 & 180.907 \\
\hline Case 6 & $1.06 \mathrm{E}+04$ & $2.11 \mathrm{E}+03$ & 5.008 & 420.801 \\
\hline Case 7 & 928.125 & 275.875 & 3.364 & 82.0008 \\
\hline Case 8 & 977.375 & 240.875 & 4.057 & 59.3639 \\
\hline
\end{tabular}

\begin{tabular}{|c|c|c|c|}
\hline \multirow{2}{*}{ US Image } & \multicolumn{3}{|c|}{ Malignant Measurement Indices } \\
\cline { 2 - 4 } & Solidity & Eccentricity & Orientation \\
\hline Case 1 & 0.6729 & 0.9953 & $1.01 \mathrm{E}+04$ \\
\hline Case 2 & 0.6984 & 0.9086 & $1.47 \mathrm{E}+04$ \\
\hline Case 3 & 0.8167 & 0.961 & 6.1294 \\
\hline Case 4 & 0.7556 & 0.9794 & -2.4143 \\
\hline Case 5 & 0.6747 & 0.9884 & -7.7571 \\
\hline Case 6 & 0.4927 & 0.941 & 21.2408 \\
\hline Case 7 & 0.8079 & 0.9895 & 1.8912 \\
\hline Case 8 & 0.7935 & 0.9785 & 3.3308 \\
\hline
\end{tabular}

The extracted features are given to the neural network to determine which type of nodule is present (malignant or benign).

\section{Conclusion}

Medical images are useful for clinical diagnosis. It is a time consuming for physicians to manually segment the thyroid nodule. From the experimental results, generation of more features may enhance the evaluation procedure accuracy.
This work gives efficient platform for researchers and scientist.

\section{References}

[1] K T Wong and Anil T Ahuja, " Ultrasound of thyroid cancer"Cancer Imaging(2005)5, 167-176DOI: 10.1102/1470-7330.2005.0110

[2] Himadri Nath Moulick, Moumita Ghosh, "Biomedical Image Processing With Nonlinear Filters" International Journal of Computational Engineering Research, Vol 03, Issue 7

[3] Rong Zhu, Yong Wang, " Application of Improved Median Filter on Image Processing" JOURNAL OF COMPUTERS, VOL. 7, NO. 4, APRIL 2012

[4] H. Mostofi and K. College, "Fast Level Set Segmentation of Biomedical Images using Graphics Processing Units, " Final year project Final year project, Department of Engineering Science, University of Oxford Oxford, 2009.

[5] C. Li, C. Xu, C. Gui, and M. D. Fox, "Distance Regularized Level Set Evolution and Its Application to Image Segmentation, " Image Processing, IEEE Transactions on, vol. 19, pp. 3243-3254, 2010.

[6] Vineetha G R1, Gopu Darshan2" Level Set Method for Image Segmentation: A Survey" IOSR Journal of Computer Engineering (IOSR-JCE) e-ISSN: 2278-0661, p- ISSN: 2278-8727Volume 8, Issue 6 (Jan. - Feb. 2013), PP 74-78

[7] R. Haralick, K. Shanmugam, and I. Dinstein, "Textural Features for Image Classification", IEEE Trans. on Systems, Man and Cybernetics, SMC-3(6):610-621, (1973)

[8] M.Seetha, I.V.Muralikrishna, B.L.Deekshatulu, B.L.Malleswari, Nagaratna, P.Hegde, "ARTIFICIALNEURAL NETWORKS AND OTHER METHODS OF IMAGE CLASSIFICATION" Journal of Theoretical and Applied Information Technology (C) $2005-2008$

[9] M. Tuceryan and A. K. Jain, Texture analysis, In The Handbook of Pattern Recognition and Computer Vision 207-248(1998).

[10] R. S. Poulsen N. G. Nguyen and C. Louis, Some new color features and their application to cervical cell classification, Pattern Recognition 164 , 401-411(1983)

[11]M. Schrder M. Schroder and A. Dimai, Texture information in remote sensing images: A case study, Workshop on Texture Analysis (1998).

[12] K. Shanmugam R. M. Haralick and I. H. Dinstein, Textural features for image classification, IEEE Transactions on Systems, Man and Cybernetics 3 (1973), 610-621

[13] J. R. Smith and F. S. Chang, Automated binary texture feature sets for image retrieval, In Proc. Int. Conf. on Acoustics, Speech, and Signal Processing (1996), 22412246.

[14] Tuan Anh Pham, " Optimization of Texture Feature Extraction Algorithm" Computer Engineering Department of Electrical Engineering, Faculty of Electrical Engineering, Mathematics and Computer Science Delft University of Technology, 2010

\section{Volume 5 Issue 1 January 2016}


[15]P. Mohanaiah, P. Sathyanarayana, L. GuruKumar, ' Image Texture feature Extraction Using GLCM Approach", International Journal of Scientific and Research Publications, Volume 3, Issue 5, May 2013

[16] Deepika Koundal1 et al., Computer-Aided Diagnosis of Thyroid Nodule:A Review, International Journal of Computer Science \& Engineering Survey (IJCSES) Vol.3, No.4, August 2012

[17] Mary C. Frates, Carol B.Benson, "Management of thyroid nodules detected at US: society of radiologists in ultrasound consensus conference statement" radiology 2005.

[18] N. Hu, D. B. Downey, A. Fenster, and H. M. Ladak, "Prostate boundary segmentation from 3d ultrasound images, "Medical Physics, vol. 30, no. 7, pp. 16481659, Jul. 2003.

[19]B. Chiu, G. H. Freeman, M. M. A. Salama, and A. Fenster, "Prostate segmentation algorithm using dyadic wavelet transform and discrete dynamic contour, "Phys. Med. Biol., vol. 49, no. 21, pp. 4943-4960, Nov. 2004.

[20] D. R. Chen, R. F. Chang, W. J. Wu, W. K. Moon, and W. L. Wu, "3-D breast ultrasound segmentation using active contour model, " Ultrasound Med. Biol., vol. 29, no. 7, pp. 1017-1026, Jul. 2003.

[21]C. Baillard C. Barillot, P. Bouthemy, "Robust adaptive segmentation of $3 \mathrm{~d}$ medical images with level sets," Institut National de Recherche en Informatique et en Automatique (INRIA), Le Chesnay Cedex, France, Tech. Rep. 4071, Nov. 2000.

[22] D. E. Maroulis, M. A. Savelonas, D. K. Iakovidis, S. A. Karkanis and N. Dimitropoulos, "Variable background active contour model for computer-aided delineation of nodules in thyroid ultrasound images, "IEEE Trans. Inf. Technol. Biomed., vol. 11, no. 5, pp. 537-543, 2007.

[23] J. C. R. Seabra, L. M. Pedro, J. F. e Fernandes, and J. M. Sanches, "A 3-D ultrasound-based framework to characterize the echo morphology of carotid plaques," IEEE Trans. Biomed. Eng., vol. 56, pp. 1442-1453, 2009. 